\title{
A GDPR-Compliant Partner Notification Service
}

Christine KAKALOU ${ }^{\mathrm{a}}$, Eleftheria POLYCHRONIDOU ${ }^{\mathrm{b}}$, Vicky DROSOU ${ }^{\mathrm{b}}$, Vlasios K. DIMITRIADIS ${ }^{\mathrm{a}}$, Thomas DERMARIS ${ }^{\mathrm{a}}$, Rafael KORDONIAS ${ }^{\mathrm{b}}$, Aris PAPAPRODROMOU ${ }^{\mathrm{b}}$, Triantafillos TSIRELIS ${ }^{\mathrm{b}}$, Christos MARAMIS ${ }^{\mathrm{a}}$, Konstantinos VOTIS $^{\mathrm{b}}$, Dimitrios TZOVARAS ${ }^{\mathrm{b}}$, Domenico SAVARINO ${ }^{\mathrm{c}}$, Manuel MAFFEO ${ }^{\mathrm{d}}$, Nedim JASIC ${ }^{e}$, Tatjana NEMETH-BLAŽIĆf ${ }^{\mathrm{f}}$, Zoran DOMINKOVIĆg ${ }^{\mathrm{g}}$, Dubravko POGLEDIĆ $^{\mathrm{g}}$, Iva JOVOVIC ${ }^{\mathrm{h}}$, Agne SIMKUNAITE-ZAZECKE ${ }^{\mathrm{i}}$, Loreta STONIENE ${ }^{\mathrm{j}}$, Antonella SAMMUT ${ }^{\mathrm{k}}$, Lella COSMARO ${ }^{\mathrm{c}}$ and Pantelis NATSIAVAS ${ }^{\mathrm{a}}$

a Institute of Applied Biosciences, Centre for Research \& Technology Hellas, Thermi, Thessaloniki, Greece

b Information Technologies Institute, Centre for Research \& Technology Hellas, Thermi, Thessaloniki, Greece

${ }^{\mathrm{c}}$ Fondazione LILA Milano - Italian League for Fighting AIDS, Milan, Italy d Arcigay, Bologna, Italy

e Croce Rossa Italiana, Rome, Italy

${ }^{\mathrm{f}}$ CIPH Croatian Institute of Public Health, Zagreb, Croatia ' Iskorak, Zagreb, Croatia

h Life Quality Improvement Organisation "Flight", Zagreb, Croatia ${ }^{\text {i } U L A C / C C D A}$ Centre for Communicable Diseases and AIDS, Vilnius, Lithuania ${ }^{\mathrm{j}}$ RPLC Republican Center for Addictive Disorders, Vilnius, Lithuania

${ }^{\mathrm{k}}$ Public Mental Health Services. Ministry for Health, Valletta, Malta

\begin{abstract}
Partner Notification processes focus on the notification of sexual partners to prevent the transmission of Sexually Transmitted Infections (STIs). The INTEGRATE Joint Action provides an integrated platform called RiskRadar, for combination prevention activities targeting STIs, including an anonymous, free and voluntary Partner Notification service. The presented service information flow ensures privacy, security and GDPR compliance which were identified as vital with similar tools. The service is available via web and mobile interfaces using a unique random code provided from authorised healthcare professionals to support privacy.
\end{abstract}

Keywords. HIV Infections, Sexually Transmitted Diseases, Contact Tracing

\section{Introduction}

Contact Tracing (CT) is the process of identifying potential infection sources and/or newly infected persons, aiming to reduce further transmission by offering testing or other preventive interventions, along with potential treatment options, depending on the specific infection per se [1]. Partner Notification (PN) is a process tightly related with

${ }^{1}$ Christine Kakalou, Institute of Applied Biosciences, Centre for Research \& Technology Hellas, 6th Km. Charilaou-Thermi Road, Thermi, Thessaloniki; E-mail: ckakalou@certh.gr. 
CT which involves voluntary notification of sexual partners of a diagnosed patient, in order to prevent further transmission of Sexually Transmittable Infections (STIs), as high prevalence rates among notified partners have been noted compared with unselectively screened individuals [2]. Obviously, PN/CT processes come with many ethical, privacy and legally related challenges, while they also come with high cost [3], [4]. Thus, digital technologies are identified as a way to improve them in a cost-effective manner [5].

The INTEGRATE Joint Action has implemented integrated activities to improve prevention, early diagnosis and linkage to care for HIV, viral hepatitis, STIs and tuberculosis in Europe. In this context, RiskRadar a publicly available web and mobile toolkit has been developed to support combination prevention, testing and linkage to care activities for the four disease areas ${ }^{2}$. This paper presents a technical solution to support the PN process in a GDPR complaint, secure and privacy-preserving manner.

\section{Methods}

The development of the PN service started with an extensive desk review to evaluate existing relevant tools and propose a prototype based on the resulting insights. Through an iterative process, the PN service was developed and tailored to identified priority groups (men who have sex with men, people who use drugs, migrants, young adults and prisoners) while ensuring its correct adaptation to all STIs. Furthermore the effective communication strategies proposed by ECDC and WHO [6], [7] were adopted and a Data Protection Impact Assessment was conducted to mitigate information security risks (e.g. service misuse, sensitive data exposure etc.) to ensure GDPR compliance.

\section{Results}

The architecture of the PN service is composed of 4 main technical components (Figure 1): a) An interface for HCPs dedicated to unique passcode generation, b) The RiskRadar backend that handles the unique random passcode generation and RiskRadar's PN service requests, c) RiskRadar's PN service frontend for index patients that can be accessed only via the generated passcodes and d) an SMS sending mechanism. To maintain privacy, log encryption and pseudonymisation of the phone numbers were applied; in addition, the phone numbers are automatically and permanently deleted from the system once the notification messages are delivered to the recipients.

RiskRadar is available to download from the official app stores ${ }^{3,4}$. Figure 2 presents the PN service information flow; healthcare professionals (HCPs) receive login credentials to use the code generator web interface in order to issue and deactivate passcodes. Once a patient receives the diagnosis, the HCP encourages the index patient to notify their past sexual partners so that they can get tested. The index patient receives a printout with the generated passcode in two formats: a serial passcode for the RiskRadar web app and a QR passcode to be scanned through the RiskRadar mobile app, along with printed instructions on how to use the PN service. The patient can then notify past sexual partners in their own time, outside of the clinical setting if they wish -

\footnotetext{
${ }^{2}$ https://integrateja.eu/riskradar/

${ }^{3} \mathrm{https}: / /$ play.google.com/store/apps/details?id=com.tdermaris.integratetoolkit

${ }^{4}$ https://apps.apple.com/app/id1469764662
} 
although contact information to reach affiliated organisations is offered should they need further support.

To use the passcode, the index patient can type/scan the passcode and then enter the mobile number(s) of the partners to be notified. They will then receive an SMS informing of potential STI exposure with information on where they can get tested. To prevent malicious use, the passcode is valid for 30 days and can be used up to 10 times - however the user is able to notify multiple partners in each round. Furthermore, every time a misuse/abuse incident is identified by the system through the auditing of the code generator database (e.g. a bot attempting to generate codes and use them to send PN messages), the healthcare professional is notified to deactivate the passcode.

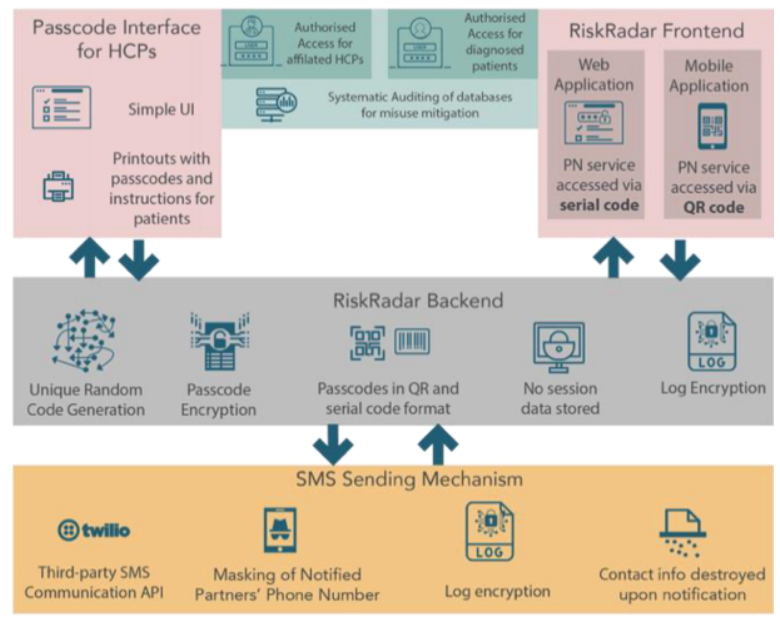

Figure 1. Partner Notification Service Architecture

\section{Discussion}

In the context of combination prevention efforts, the proposed service offers free, voluntary, anonymous Partner Notification and thus promotes patient empowerment and reduces stigmatisation. Concerning the service's strengths, its architecture ensures the security, privacy and confidentiality of all stakeholders involved; Furthermore, the HCPauthorised access hinders misuse attempts. We expect that the enhanced security and privacy of the service will boost its acceptance especially among vulnerable populations. Regarding limitations of this work, providing a printout of the instructions and passcodes is an obvious liability that could expose a patient's diagnosis. Another limitation is the prerequisite of a patient knowing the phone number of past sexual partners, which may be problematic especially for people engaging in casual sex. A possible workaround would be the integration of the PN service to popular dating mobile apps. Additionally, detecting a misuse of the PN service could be challenging, even with the authorisation process that is currently in place. Finally, the acceptability, usability and increased preference to the proposed PN tool over other solutions needs to be validated through a robust evaluation phase by engaging members of the community. 

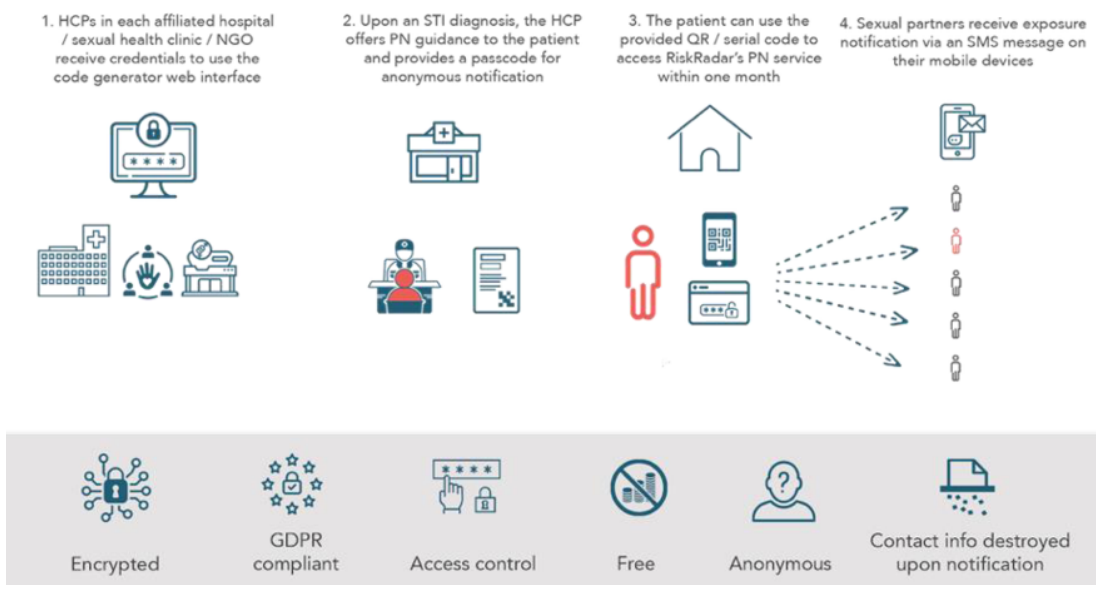

Figure 2. Information flow for Partner Notification service.

\section{Conclusions}

The presented PN service emphasizes the need to efficiently and securely notify sexual partners for a potential transmission of STIs. While the presented platform has not yet been pilot tested, we argue that such an approach could provide an alternative to traditional costly and ethically, legally and privacy-challenging PN approaches.

\section{Acknowledgements}

This work has received funding from the European Union for the INTEGRATE Joint Action within the framework of the Third Health Programme (grant number 761319).

\section{References}

[1] European Centre for Disease Prevention and Control. Public health guidance on HIV, hepatitis B and C testing in the EU/EEA -. 2018.

[2] Wang AL, Peng RR, Tucker JD, Cohen MS, Chen XS. Partner notification uptake for sexually transmitted infections in China: A systematic literature review. Sexually Transmitted Infections. NIH Public Access. 2012;88:386-93.

[3] Johnson BL, Tesoriero J, Feng W, Qian F, Martin EG. Cost Analysis and Performance Assessment of Partner Services for Human Immunodeficiency Virus and Sexually Transmitted Diseases, New York State, 2014. Health Serv Res. 2017 Dec;52(2):2331-42.

[4] Gibbs J, Gkatzidou V, Tickle L, Manning SR, Tilakkumar T, Hone K, Ashcroft RE, Sonnenberg P, Sadiq ST, Estcourt CS. 'Can you recommend any good STI apps?'A review of content, accuracy and comprehensiveness of current mobile medical applications for STIs and related genital infections. Sexually transmitted infections. 2017 Jun 1;93(4):234-5.

[5] Althaus CL, Turner KM, Mercer CH, Auguste P, Roberts TE, Bell G, Herzog SA, Cassell JA, Edmunds WJ, White PJ, Ward H. Effectiveness and cost-effectiveness of traditional and new partner notification technologies for curable sexually transmitted infections: observational study, systematic reviews and mathematical modelling. Health technology assessment (Winchester, England). 2014;18(2):1-viii.

[6] European Centre for Disease Prevention and Control. Utilising social media to support HIV/STI prevention: evidence to inform a handbook for public health programme managers. 2017. 
[7] WHO. Guidelines on HIV self-testing and partner notification. Supllement to Consolidated Guildelines on HIV Testing Services. World Health Organization. 2016. 